\title{
EL ALIVIO DEL DOLOR EN EL PARTO. EMPODERAMIENTO Y VULNERABILIDAD DE LAS MUJERES EN LA TOMA DE DECISIONES. ESTUDIO CUALITATIVO ${ }^{1}$
}

\author{
Josefina Goberna-Tricas ${ }^{1}$, Ainoa Biurrun-Garrido ${ }^{2,3}$ \\ Correo electrónico: jgoberna@ub.edu
}

1. Departamento de Enfermería de Salud Pública, Salud Mental y Maternoinfantil. Facultad de Medicina y Ciencias de la Salud. ADHUC. Centro de Investigación en Teoría, Género y Sexualidad. Universitat de Barcelona.

2. Campus Docent Sant Joan de Déu. Barcelona.

3. Hospital del Mar. Barcelona.

Recibido: 24/11/2019 Aceptado: 17/01/2020

\footnotetext{
${ }^{1}$ La investigación que ha dado lugar a estos resultados ha estado impulsada por el Proyecto de Investigación: «Vulnerabilidad en el pensamiento filosófico femenino. Contribuciones al debate sobre emergencias presentes», PGC2018-094463-B-100(MCIU/AEI/FEDER, UE). Se han reanalizado algunas de las entrevistas realizadas por la segunda autora en su tesis doctoral.
} 


\section{RESUMEN}

\section{Introducción}

En nuestro entorno, el uso de la epidural para el alivio del dolor en el parto se encuentra muy extendido. Actualmente, se está produciendo un cambio de actitud que pretende avanzar hacia una atención menos intervencionista, ofreciendo métodos no farmacológicos para reducir el dolor antes de aplicar técnicas espinales, siempre que la mujer no haya proporcionado otras indicaciones precisas.

\section{Objetivo}

Profundizar en el proceso de toma de decisiones en relación con el abordaje del dolor en el parto para conocer los elementos de vulnerabilidad presentes en la toma de decisiones e indagar en las demandas dirigidas hacia los profesionales sanitarios.

\section{Metodología}

Estudio mediante metodología cualitativa con una perspectiva teórico-metodológica fenomenológica. La técnica utilizada para la obtención de información fue la entrevista individual semiestructurada. El estudio se llevó a cabo en Cataluña. Las participantes fueron 17 mujeres mayores de edad con embarazos de bajo riesgo.

\section{Resultados}

Tres categorías han sido identificadas tras el análisis: (a) La cumplimentación del plan de parto: una aproximación a la decisión, (b) Los fundamentos de la decisión: recursos y barreras, y (c) El abordaje del dolor en el trabajo de parto: elementos de vulnerabilidad.

\section{Conclusiones}

Las mujeres no siempre toman decisiones firmes en el momento de cumplimentar el plan de parto; las decisiones manifestadas pueden ser declaraciones de intenciones. En el momento del parto, el dolor percibido puede vivirse como una amenaza frente a la cual se sienten vulnerables. El rol de las matronas y otros profesionales sanitarios puede ayudar a la resolución de esta sensación de vulnerabilidad.

Palabras clave: parto; dolor; investigación cualitativa; plan de parto; bioética; decisiones informadas. 


\section{ABSTRACT}

\section{Introduction}

In Spain the use of epidurals for pain relief during childbirth is widespread. Currently, we can observe a progressive change in attitude towards a less interventionist care and the use of nonpharmacological methods to reduce pain, unless the woman has left instructions otherwise.

\section{Aims}

To explore the decision-making process concerning pain relief methods in childbirth in order to detect the elements of vulnerability that are present in said process and examine the demands directed towards healthcare professionals.

\section{Methodology}

This study has applied a qualitative methodology with a phenomenological theoreticalmethodological approach. The technique used to obtain information was the semistructured individual interview. The study was carried out in Catalonia. The participants were 17 adult women with low-risk pregnancies.

\section{Results}

Three categories have been identified after the analysis: (a) Completion of the birth plan: an approach to the decision; (b) The rationale of the decision: resources and barriers; and (c) The approach to pain in childbirth: elements of vulnerability.

\section{Conclusions}

Women do not always make firm decisions when completing the birth plan; The decisions expressed may be statements of intent. At the time of delivery, the perceived pain can be experienced as a threat that makes them feel vulnerable. The role of midwives and other healthcare professionals is to help work out this sense of vulnerability.

Keywords: Birth; Pain; Qualitative Research; Birth Plan; Bioethics; Informed Consent. 


\section{INTRODUCCIÓN}

La percepción del dolor en el parto constituye un fenómeno complejo, subjetivo y multidimensional que no se construye únicamente a partir de los componentes sensoriales, sino que también comprende aspectos emocionales, motivacionales y cognitivos (Van der Gucht y Kiara, 2015).

El Protocolo de asistencia al parto y al puerperio y de atención al recién nacido (Generalitat de Catalunya, 2003) indica que la utilización de analgesia durante el parto es mucho más que una indicación de tipo médico, ya que representa un índice de calidad muy importante en la prestación asistencial a la mujer embarazada. En nuestro entorno, el uso de la epidural como método de alivio del dolor en el parto se popularizó en España a finales del siglo pasado, siendo Cataluña una de las Comunidades Autónomas pioneras en la oferta de este tipo de anestesia, tanto en los centros de financiación pública como privada. En el año 2003, se realizó anestesia epidural, en Cataluña, al 82\% de las mujeres que dieron a luz por parto vaginal y cesárea (Sabaté et al., 2006).

A partir de 2008, las propuestas del Ministerio de Sanidad y Consumo en la Estrategia de Atención al Parto Normal y, posteriormente, en el año 2010, la Guía de Práctica Clínica sobre la Atención al Parto Normal reconocen que «la atención al parto se debe realizar bajo el concepto general de que el nacimiento es un proceso fisiológico en el que sólo se debe intervenir para corregir desviaciones de la normalidad». Actualmente, se está produciendo un cambio de actitud y se pretende avanzar hacia una atención menos intervencionista, ofreciendo métodos no farmacológicos para reducir el dolor antes de aplicar técnicas espinales, siempre que la mujer no haya proporcionado otras indicaciones precisas.

Entre los objetivos de la Estrategia de atención al parto normal se encuentra la incorporación activa de las gestantes en la toma de decisiones informadas en la atención al parto, así como ofrecer cuidados individualizados basados en la evidencia científica, y adaptados a las necesidades de cada mujer. Con el objetivo de hacer efectiva la participación de las mujeres en la toma de decisiones durante la atención al parto, se han elaborado y promocionado los denominados «planes de parto». El Ministerio de Sanidad, Política Social e Igualdad (2012) ha desarrollado un modelo de plan de parto que se propone como guía para que cada centro asistencial desarrolle el suyo propio, de acuerdo 
con sus recursos y disponibilidad. El plan de parto recoge, entre otros aspectos, la expresión del deseo de las mujeres en cuanto al método deseado para el alivio del dolor.

El reconocimiento del derecho de la mujer a cumplimentar el plan de parto y tomar decisiones relacionadas con su asistencia implica un reconocimiento del respeto al principio de autonomía en la toma de decisiones. La toma de decisiones y su resultado las elecciones informadas - son un buen indicador de una atención centrada en la mujer que se fundamenta en un respeto a las decisiones tomadas tras un proceso de información y diálogo entre los profesionales (matronas y obstetras) y las mujeres y sus parejas.

El reconocimiento del principio de autonomía de los pacientes es un derecho reconocido y respaldado por el marco legal vigente en España mediante la Ley 41/2002, de autonomía del paciente. En el ámbito europeo, entre los años 1993 y 1998, se trabajó en la posibilidad de formular un «principalísimo europeo» que propone considerar junto al principio de autonomía los principios de dignidad, integridad y vulnerabilidad; este esfuerzo culminó en la llamada Declaración de Barcelona (2000) y a partir de entonces el principio de vulnerabilidad se incluyó en la Declaración de la UNESCO (2013) sobre bioética y Derechos Humanos tanto en Europa como en el mundo anglosajón y en América Latina. De este modo, a partir de la década de los noventa del siglo XX, el concepto de vulnerabilidad se inscribe cada vez con mayor fuerza en el ámbito sanitario.

Briscoe et al (2016) realizan un análisis del concepto de vulnerabilidad durante embarazo, parto y puerperio identificando que las mujeres se sienten vulnerables cuando experimentan una sensación de amenaza desde una perspectiva física, psicológica o social; el nivel de amenaza se expresa mediante la interacción entre la posibilidad de soluciones reparativas frente a dicha amenaza y las barreras percibidas para su resolución. El afrontamiento del dolor en el parto puede ser vivido por las mujeres como un factor de vulnerabilidad.

La matrona es el profesional de referencia en el seguimiento del embarazo normal en el Sistema Sanitario Catalán. Debido a su contacto más directo con las mujeres durante embarazo, parto y postparto, tiene un papel crucial en el proceso de información y asesoramiento del cumplimiento del plan de parto, así como en el correcto abordaje de la elección y toma de decisiones en relación con el método de alivio del dolor. Por ello, en este trabajo, nos hemos fijado como objetivo principal indagar en el proceso de toma de decisiones de las mujeres en relación con el abordaje del dolor en el parto. Además, 
pretendemos profundizar en este proceso para conocer los elementos de vulnerabilidad presentes durante la toma de decisiones, así como indagar en las demandas dirigidas hacia los profesionales que darán asistencia al parto (matronas y obstetras).

\section{METODOLOGÍA}

\section{Diseño del estudio}

Esta investigación se realizó mediante uso de metodología cualitativa. La metodología cualitativa reconoce a la persona y a su contexto, las razones que existen detrás de los hechos y cómo las personas dan sentido a sus propias acciones. Se utilizó la perspectiva teórico-metodológica de la fenomenología (Matua, 2015) para investigar la percepción de las mujeres sobre «la toma de decisiones en relación con el método de alivio del dolor durante la atención al parto, con el fin de averiguar si se sienten vulnerables y que barreras o soluciones reparativas encuentran, así como profundizar en las demandas que dirigen a los profesionales en el proceso de toma de decisiones».

\section{Ámbito de estudio}

El estudio se llevó a cabo en Cataluña, donde existen un total de 9 regiones sanitarias que atienden mujeres durante su gestación y parto. El 99\% de los partos que se asisten en este territorio se producen en el ámbito hospitalario (Escuriet et al., 2015).

\section{Participantes del estudio}

Las participantes fueron mujeres mayores de edad con embarazos normales o de bajo riesgo que dieron a luz entre los años 2012 y 2014 en Cataluña. Las primeras participantes se seleccionaron gracias a contactos personales de una de las investigadoras con mujeres que habían sido madres recientemente, y gracias a la técnica de bola de nieve se amplió la búsqueda de nuevas informantes que coincidieran con criterios teóricos previamente establecidos. Se ha buscado la máxima variabilidad a partir de los siguientes criterios: edad, paridad (primíparas y multíparas), nivel de estudios (básicos o secundarios y universitarios), ámbito de residencia (zona rural o urbana), y tipo de método de alivio de dolor deseado y manifestado en el plan de parto (métodos no farmacológicos o utilización de anestesia epidural). Una vez iniciadas las entrevistas, se comprobó que el ámbito de residencia (zona rural o urbana) no daba lugar a diferencias en los discursos de las participantes. Se realizaron un total de 17 entrevistas. La saturación teórica de los datos 
fijó el tamaño final de la muestra. El perfil de las mujeres entrevistadas se puede consultar en la Tabla 1. Los nombres usados en este artículo no son los reales, con el fin de proteger la confidencialidad de las participantes.

Tabla 1. Perfil de las mujeres entrevistadas

\begin{tabular}{|c|c|c|c|c|c|}
\hline $\begin{array}{l}\text { Nombre } \\
\text { (ficticio) }\end{array}$ & Edad & $\begin{array}{c}\text { Nivel } \\
\text { Educación }\end{array}$ & Paridad & $\begin{array}{l}\text { Tipo de analgesia } \\
\text { elegida e indicada } \\
\text { en el plan de parto }\end{array}$ & $\begin{array}{c}\text { Tipo de } \\
\text { analgesia } \\
\text { en el } \\
\text { parto }\end{array}$ \\
\hline Delia & 21 & Primaria & Primípara & $\begin{array}{c}\text { Método no } \\
\text { farmacológico }\end{array}$ & $\begin{array}{c}\text { Anestesia } \\
\text { epidural }\end{array}$ \\
\hline Esther & 29 & Primaria & Tercípara & Anestesia epidural & $\begin{array}{c}\text { Anestesia } \\
\text { epidural }\end{array}$ \\
\hline Susana & 29 & Primaria & Secundípara & Anestesia epidural & $\begin{array}{l}\text { Anestesia } \\
\text { epidural }\end{array}$ \\
\hline Isabel & 42 & Secundaria & Primípara & Anestesia epidural & $\begin{array}{c}\text { Anestesia } \\
\text { epidural }\end{array}$ \\
\hline Jimena & 38 & Secundaria & Primípara & Anestesia epidural & $\begin{array}{c}\text { Anestesia } \\
\text { epidural } \\
\text { (cesárea) }\end{array}$ \\
\hline Muriel & 32 & Universitaria & Primípara & $\begin{array}{c}\text { Método no } \\
\text { farmacológico }\end{array}$ & $\begin{array}{c}\text { Anestesia } \\
\text { epidural }\end{array}$ \\
\hline Mercedes & 22 & Primaria & Primípara & $\begin{array}{l}\text { Método no } \\
\text { farmacológico }\end{array}$ & $\begin{array}{c}\text { Anestesia } \\
\text { epidural }\end{array}$ \\
\hline Sònia & 19 & Primaria & Primípara & $\begin{array}{l}\text { Método no } \\
\text { farmacológico }\end{array}$ & $\begin{array}{c}\text { Anestesia } \\
\text { epidural }\end{array}$ \\
\hline Valentina & 25 & Secundaria & Secundípara & Anestesia epidural & $\begin{array}{c}\text { Anestesia } \\
\text { epidural }\end{array}$ \\
\hline Margarita & 35 & Universitaria & Tercípara & Anestesia epidural & $\begin{array}{c}\text { Anestesia } \\
\text { epidural }\end{array}$ \\
\hline Cristina & 29 & Universitaria & Primípara & $\begin{array}{c}\text { Método no } \\
\text { farmacológico }\end{array}$ & $\begin{array}{c}\text { Anestesia } \\
\text { epidural }\end{array}$ \\
\hline Lourdes & 33 & Universitaria & Primípara & $\begin{array}{c}\text { Método no } \\
\text { farmacológico }\end{array}$ & $\begin{array}{c}\text { Sin } \\
\text { anestesia } \\
\text { epidural }\end{array}$ \\
\hline Vicenta & 30 & Secundaria & Secundípara & $\begin{array}{c}\text { Método no } \\
\text { farmacológico }\end{array}$ & $\begin{array}{c}\text { Sin } \\
\text { anestesia } \\
\text { epidural }\end{array}$ \\
\hline Rosalía & 26 & Primaria & Primípara & Anestesia epidural & $\begin{array}{c}\text { Anestesia } \\
\text { epidural }\end{array}$ \\
\hline Gisela & 35 & Primaria & Secundípara & Anestesia epidural & $\begin{array}{c}\text { Anestesia } \\
\text { epidural }\end{array}$ \\
\hline Carolina & 30 & Primaria & Secundípara & $\begin{array}{c}\text { Método no } \\
\text { farmacológico }\end{array}$ & $\begin{array}{c}\text { Anestesia } \\
\text { epidural }\end{array}$ \\
\hline Adelaida & 39 & Universitaria & Secundípara & $\begin{array}{l}\text { Método no } \\
\text { farmacológico }\end{array}$ & $\begin{array}{c}\text { Anestesia } \\
\text { epidural } \\
\text { para } \\
\text { cesárea }\end{array}$ \\
\hline
\end{tabular}


A partir de la información aportada por las primeras entrevistas, las investigadoras se pusieron en contacto con el resto de las mujeres, a las que se les explicó el objetivo del estudio, se les pidió su consentimiento para realizar y grabar las entrevistas y se les garantizó la confidencialidad de la información obtenida.

\section{Técnica de recogida de datos}

La técnica utilizada para la obtención de información fue la entrevista individual Se contó con una guía de entrevista con el objetivo de asegurar que se respondía a todos los aspectos fijados en los objetivos planteados. Sin embargo, las preguntas no se realizaron en un orden predeterminado con el objetico de no interrumpir las narrativas de las mujeres entrevistadas. Al inicio de la entrevista, se pidió a las mujeres que explicaran su experiencia y que indicaran qué decisiones habían tomado relacionadas con la asistencia sanitaria y el alivio del dolor. El guion de las entrevistas puede consultarse en la Tabla 2.

\section{Tabla 2. Guion de las entrevistas}

\section{Preguntas generales:}

- ¿Cómo has vivido el parto? Explícame tu experiencia.

- Durante tu embarazo. ¿Cómo pensabas que iba a ser tu parto? ¿Coincidieron tus expectativas con la realidad?

- ¿Cómo te sentiste durante el parto? ¿Cómo viviste el dolor?

\section{Preguntas específicas:}

- ¿Tomaste decisiones relacionadas con el tipo de atención al parto? ¿Cuáles?

- ¿Y sobre el alivio del dolor? ¿Qué papel jugaste tú en estas decisiones? ¿Y tu pareja? ¿Y el personal sanitario? ¿Se aplicaron estas decisiones?

- ¿Dónde obtuviste la información relacionada con los métodos de alivio del dolor? ¿Habrías necesitado más información? ¿De qué tipo?

- ¿Consideras que se respetó tu intimidad? ¿Cómo? ¿Te sentiste respetada?

- ¿Cómo viviste el trato con los profesionales? ¿Puedes identificar qué tipo de profesionales te atendieron durante el parto? ¿Qué opinión te formaste? ¿Qué rol ocuparon en las decisiones y el afrontamiento del dolor?

- ¿Qué crees que te aportaron los profesionales sanitarios en las decisiones que tomaste durante la atención al parto? 
La duración de las entrevistas osciló entre los 45 y los 90 minutos. Las entrevistas se realizaron en un lugar donde las participantes se sintieran cómodas; algunas en los domicilios de las mujeres, otras, en lugares públicos tales como cafeterías o salas de espera de centros de atención primaria.

Antes de empezar la entrevista, se informó a las mujeres de los objetivos del estudio, la preservación del anonimato y la confidencialidad de los datos. Todas las entrevistas fueron grabadas en audio y después se procedió a la transcripción de las mismas a fin de facilitar el posterior análisis. Además, se utilizó un diario de campo durante las entrevistas como instrumento de recogida de las reflexiones y observaciones de las investigadoras durante todo el proceso.

\section{Análisis de los datos}

Después de una lectura en profundidad del texto, se procedió al análisis del contenido con codificación y categorización de acuerdo con las tres fases definidas por Taylor y Bogdan (1987): En primer lugar, la fase de descubrimiento o preanálisis, en la que, después de una relectura de los datos, se procedió a una identificación preliminar de temas y a una búsqueda de temas emergentes. En segundo lugar, se pasó a la codificación, mediante la cual se identificaron las categorías, se procedió a una segmentación del texto de acuerdo con las categorías establecidas, se comprobaron los datos sobrantes y se refinó el análisis a la vista del conjunto. Finalmente, en la tercera fase, se procedió a una relativización de los datos confrontándolos con el contexto en el que se habían obtenido y comparándolos con la teoría para crear un marco explicativo. Las discrepancias que emergieron fueron consensuadas entre las dos investigadoras. Se contó con la ayuda del programa Atlas-Ti v.7.

\section{Aspectos éticos}

Este estudio cuenta con la autorización de la Comisión de Bioética de la Universidad de Barcelona (IRB00003099) y el consentimiento informado de cada mujer. Se garantizó la confidencialidad de datos y se usaron seudónimos en las transcripciones. 


\section{RESULTADOS}

Los resultados del estudio han permitido comprender la experiencia vivida por las mujeres en relación con la toma de decisiones sobre el método de alivio del dolor a lo largo del proceso del parto, identificando elementos de vulnerabilidad, así como aquellos aspectos en que la intervención de los profesionales sanitarios (matronas y obstetras) puede ayudar a superar estas situaciones.

Presentaremos esta información ordenada a partir de los tres temas identificados como centrales en el análisis: (a) La cumplimentación del plan de parto: una aproximación a la decisión, (b) Los fundamentos de la decisión: recursos y barreras, y (c) El abordaje del dolor en el trabajo de parto: elementos de vulnerabilidad.

\section{La cumplimentación del plan de parto: una aproximación a la decisión}

Todas las mujeres indican que la decisión referente a como querrán afrontar el dolor durante el parto y por qué método quieren optar para aliviarlo les compete a ellas y se sienten capacitadas para tomar una decisión informada en el momento de cumplimentar el plan de parto. Algunas de las mujeres entrevistadas afirman que desean un alivio eficaz del dolor tan pronto como sea posible y optan por pedir la anestesia epidural, como observamos en los siguientes casos:

Esther (tercípara): Sí, yo tenía claro que en este parto quería la epidural porque ya la utilicé en mi primer hijo; en cambio en el segundo no me la pusieron y lo pasé muy, muy mal.

Gisela (secundípara): Sí, sí, yo quería la epidural. Yo soy de la liga antidolor, como digo yo.

En otros casos, nos encontramos con mujeres que quieren evitar la epidural, mujeres con una alta percepción de sus propias capacidades para afrontar el dolor del parto que consideran que pueden prescindir de ayudas farmacológicas, como podemos comprobar en el caso de esta primípara:

Vicenta (primípara): Yo tenía muy claro que no quería la epidural... Bueno, yo soy una persona «muy mental» y me gusta tenerlo todo bajo control. 
Un importante grupo de mujeres, a pesar de reconocer que son ellas quienes deben decidir en relación con el método usado para el alivio del dolor, reconocen que la decisión tomada en el momento de cumplimentar el plan de parto se considera como una manifestación de intenciones, una decisión provisional, que no se convertirá en definitiva hasta el momento del parto. En este sentido, son frecuentes las frases del tipo: «me gustaría», «he pensado», pero siempre considerando que será después, en el momento del parto y tras algunas contracciones cuando podrán tomar la decisión definitiva.

Rosalía (primípara): Yo, aquí, en las clases, siempre había dicho que quería probar sin epidural, aguantar, porque mi madre siempre me decía: «si nosotras hemos aguantado, tú puedes aguantar». Vale, y, lo dije: «Voy a aguantar todo lo que pueda», pero yo no me esperaba los dolores que tuve y con las contracciones... pedi la epidural

En ciertos casos, algunas mujeres incluso prefieren no elaborar el documento «escrito» de plan de parto, temerosas de que los profesionales puedan entender estas intenciones, pendientes de confirmación, como decisiones en firme. Consideran que la cumplimentación del plan de parto puede convertirlas en vulnerables frente a los profesionales. Prefieren expresar su decisión durante el trabajo de parto, dependiendo de cómo se desarrolle este. Podemos comprobarlo en el siguiente caso:

Carolina (secundípara). No quería llevar nada escrito porque después ya no puedes cambiar de opinión.

\section{Los fundamentos de la decisión: recursos y barreras}

La fundamentación en esta disparidad en la toma de posición en relación con el método de alivio del dolor debemos buscarla, fundamentalmente, en la comprensión que las mujeres elaboran en relación con el parto, así como el significado que le otorgan al dolor. Las mujeres parten de una concepción personal del significado del parto, del dolor y de su asistencia, que han integrado en su imaginario. Esta preconceptualización la han elaborado a partir de sus valores y creencias; con ello las mujeres se forman unas expectativas en relación con cómo imaginan que será su parto, cuáles son sus capacidades y qué tipo de ayuda desean o necesitan para abordar el dolor.

En el caso de las mujeres multíparas, en la toma de decisiones en relación con el método preferido para el alivio del dolor, es fundamental la propia experiencia personal. 
Las mujeres que han dado a luz anteriormente tienen una idea bastante clara acerca de sus preferencias fundamentada en la experiencia vivida en los partos anteriores. Como en el caso de esta mujer que ya tenía dos hijos anteriores:

Raquel (tercípara): Bueno, yo ya era experta en la materia, por los otros niños que he tenido.

La experiencia de otras mujeres de la familia también influye en el significado del dolor que la propia mujer construye. Las mujeres cercanas como madre, hermanas... son fuente común de información e influyen en la decisión a adoptar.

\section{Isabel (primípara): Con mi hermana yo me sentía informada.}

Nos hemos interesado por conocer otras fuentes de información en las que las mujeres han fundamentado sus decisiones y en conocer qué importancia tiene para ellas tener información sobre los métodos de alivio del dolor. Las mujeres obtienen la información necesaria para la toma de decisiones sobre el tipo de método para el alivio del dolor de diversas fuentes. En este sentido, consideran importante a los profesionales sanitarios; la información obtenida es considerada por las mujeres entrevistadas, como buena, fiable y de calidad; tanto aquella facilitada por la matrona de atención primaria durante el seguimiento del embarazo como la obtenida en la visita preparto que las embarazadas realizan en el hospital de referencia entre las 32 y 34 semanas de gestación:

\footnotetext{
Adelaida (secundípara): En la consulta del hospital me dieron toda la información, aquí en el ambulatorio quizás no fue tan exhaustiva, pero fue completa.
}

Hemos indagado en la importancia otorgada a la información recibida en los cursos de educación maternal. En general, los cursos son bien valorados, fundamentalmente por las clases de ejercicios físicos y por poder compartir experiencias con otras mujeres, pero no tanto como fuente de información para la toma de decisiones. Un ejemplo de ello podemos observarlo en el caso de esta mujer:

Lourdes (primípara): Como yo ya había leído bastante, las clases me sonaban repetitivas. Lo que más me gustó fueron las clases de gimnasia con la pelota. Son clases largas, vienen a durar dos horas, pero están bien 
Incluso, en algunos casos, la reflexión posterior, durante los primeros meses del posparto, lleva a algunas mujeres a considerar que las clases de educación maternal a las que acudieron durante el embarazo no fueron suficientemente objetivas, habiéndolas inducido a pensar que el parto iba a ser más fácil de lo que luego resultó y entienden que se les ha encaminado hacia un modelo de atención que no cumple con sus expectativas. Algunas mujeres consideran que en los cursos de educación maternal, a veces, se fomentan falsas expectativas, como en el siguiente caso:

Cristina (primípara): En el curso de educación maternal se explicó mucho el tema del parto natural y al final piensas..., es lo que hemos de hacer, es lo mejor..., pero en cambio no se acaba de explicar bien el tema de la epidural. No me explicaron claramente cuáles eran los riesgos y las ventajas, no me dieron ninguna información escrita para que yo lo pudiera valorar tranquilamente en casa. Para mi este hubiera sido el punto más importante.

Los medios de comunicación, fundamentalmente Internet, constituyen una potente fuente de información a la que acuden la mayoría de las mujeres entrevistadas. Esta red de información ayuda a las mujeres reafirmarse en la elección tomada:

Isabel (primípara): Si tenía dudas, acudía a Internet.

Todas las mujeres entrevistadas conocen la epidural como medio de alivio del dolor, pero, en general, su conocimiento de otras técnicas o métodos no es generalizado, como en el caso de esta primípara, que comentaba:

Cristina (primípara): Yo solamente conocía la bañera, pero no me convencía. En la bañera no flotas. A mí me gusta mucho nadar, pero en la bañera... Ahora embarazada me muevo mal y sería incapaz de sentirme cómoda... No, no conozco ningún otro método... Quizás las pelotas, pero no me convence.

En cambio, aquellas mujeres que se muestran muy seguras de querer una atención sin intervenciones farmacológicas suelen conocer y usar de forma habitual terapias alternativas o completarías como podemos comprobar en el siguiente caso:

Vicenta (primípara): Sobre todo es importante la preparación psicológica... Yo no quería cosas farmacológicas; si durante siglos las mujeres han aguantado, porque ponerse sustancias que no son necesarias... Durante el 
embarazo yo hice reiki, tomé flores de Bach... Hice de todo, pero en el parto todo ha sido todo muy fácil.

\section{El abordaje del dolor en el proceso del parto: elementos de vulnerabilidad}

Las mujeres afirman que es en el momento del parto cuando las decisiones se llevan a la práctica y cuando el soporte de los profesionales resulta fundamental. Las mujeres piden una atención personalizada, quieren que los profesionales sanitarios (matronas, obstetras $\mathrm{y}$ anestesistas) las informen sobre el avance del parto, colaboren con ellas, las aconsejen sobre lo que puede resultar mejor y lleguen a un consenso y respeto de las decisiones. Así fue en el caso de una primípara que comentaba:

Cristina (primípara): La matrona que me atendió, al ver que me costaba dilatar, me aconsejo la epidural, actuó como una profesional que conoce su trabajo y seguir su recomendación me fue muy bien

Algunas mujeres reconocen que las expectativas forjadas durante el embarazo no coinciden con la realidad en el trabajo de parto. Si la realidad rompe con las expectativas previas o con la decisión previamente manifestada, puede generar cierta frustración y hacer que se sientan vulnerables. Un ejemplo de ello podemos encontrarlo en el siguiente caso de una secundípara que tuvo un parto natural con el primer hijo y con el segundo aceptó una anestesia epidural debido a que se alargó mucho el periodo de dilatación, la mujer explica que «se dejó» convencer por la matrona para que le aceleraran el proceso:

Adelaida (secundipara): En el segundo, ahora me arrepiento de alguna decisión que tomé, porque fui al hospital con contracciones, todo normal... Con la niña todo había ido muy rápido y en este segundo pasé muchas horas... y el niño no bajaba, no se encajaba, yo tenía muchas contracciones, cada 5 minutos, pero no bajaba y me puse muy nerviosa... Tantas horas... Y acepté agilizar el parto, pero para agilizarlo me tenían que poner oxitocina y anestesia epidural... y en principio yo no la quería, pero al final, tuve un momento..., bueno, de debilidad... y dije bueno, que me la pongan, si me la tienen que poner..., bien, que hagan lo que quieran..., y claro esto no era lo que yo quería y ahora le doy vueltas... y pienso da igual que te doliera, debía haber aguantado un poco más porque hubiera llegado el momento en que el niño se habría encajado y habría nacido... pero bueno fue así y a mí me ha 
quedado la idea de que todo fue porque yo dejé que intervinieran en este parto. Si yo hubiera dejado hacer a mi cuerpo, habría tenido un parto natural.

En este caso, la actitud de los profesionales es vista por esta mujer, en su análisis tras el parto, como una dificultad, como un aspecto negativo que la ha dejado inerme y vulnerable, ya que no le ha ayudado a cumplir con aquello que hubiera deseado para su parto. Esta frustración se manifiesta de forma más evidente en aquellas mujeres que se han posicionado firmemente en una decisión, tanto a favor como en contra del uso de epidural. En el caso de las mujeres que simplemente expresan sus preferencias, su declaración de intenciones como provisionales, confían más en el consejo de los profesionales y consideran la información y consejos recibidos durante el parto como elementos de seguridad que las refuerzan en la toma de decisiones. Incluso cuando cambian de opinión en relación con aquello manifestado en el plan de parto, estas mujeres aceptan bien el cambio si se acompaña de explicaciones y consejos de los profesionales. Esto lo podemos comprobar en el siguiente caso de una primípara que quería anestesia epidural, pero, cuando la solicitó, el expulsivo estaba muy próximo y se la desaconsejaron:

Rosalía (primípara) digo: « ¿Me vais a pinchar ya?». Me miraron... Yo solo quería que me pincharan, que me pusieran la anestesia... Entró la anestesista y le preguntó a la matrona: «¿De cuánto está dilatada?» «De 8». Y dice: «Pues yo me voy» « ¿Cómo que te vas?». Dice: «Mujer, es que ya no vale la pena pincharte. Piénsatelo». Dice: «Mira, mientras yo te pincho y no te pincho, has terminado de dilatar». Dice: «Yo si quieres, me quedo aquí contigo, si vemos que vas muy lenta, yo te ayudo». «Bueno, vale». Se quedó allí conmigo, y es verdad, a la media hora, me volvió a mirar y ya había dilatado.

\section{DISCUSIÓN}

Como hemos comentado, nuestro estudio ha sido realizado en Cataluña (España), donde existe una amplia cultura de uso de epidural que en los últimos años se está modificando. Sin embargo, de acuerdo con nuestros resultados, este cambio se ha forjado fundamentalmente en el ideario del ámbito sanitario, pero no ha cuajado, del mismo 
modo, en el imaginario social. Muchas mujeres siguen concibiendo el parto como una situación de riesgo, un momento especialmente vulnerable y consideran que usar un potente método de alivio del dolor resulta imprescindible. En este sentido, Coxon et al. (2014) reconocen que la influencia de la familia y los amigos, así como las creencias, valores, experiencias pasadas y valores culturales tienen una influencia muy fuerte en la elección y la toma de decisiones para las mujeres durante el embarazo y el parto.

Hemos comprobado como un grupo importante de mujeres llegan al parto con dudas en relación con el tipo de método a usar para el alivio del dolor. Para ellas el plan de parto constituye solo una declaración de intenciones e incluso en ocasiones no quieren cumplimentarlo por temor a no poder cambiar de opinión. Cabe remarcar que Welsh (2014) y Aragon (2013) explican que «Plan de parto» resulta un término inadecuado, ya que un parto es algo imposible de planear. Por ello se plantean otros nombres para dicho documento tales como: «Guía para el parto» (Brauer, 2016) o «preferencias para el parto» (Whirtford, 2014; Gijon, 2016).

Existen mujeres que desean una atención con menos intervenciones farmacológicas y consideran la posibilidad de prescindir de la anestesia epidural. Sin embargo, muchas veces, esta decisión se modifica y su idea previa de «intentar» un parto «más natural» sin epidural cambia cuando llegan las contracciones. Consistente con los resultados de nuestro estudio, los datos de Lally et al. (2014) explican que a muchas mujeres les resultó difícil determinar cómo podrían realmente tomar decisiones sobre el parto cuando todavía no estaban del todo seguras de qué esperar. Withford et al. (2014) observaron que muchas de las mujeres expresaron cierta renuencia a hacer planes dada la imprevisibilidad del trabajo de parto.

Para la mayoría de las mujeres resulta fundamental el soporte de las matronas y otros profesionales sanitarios durante el parto y piden información y consejos personalizados sobre la mejor forma de abordar el parto y la mejor manera de aliviar el dolor. El modelo de «toma de decisiones compartidas» enfatiza la necesaria interrelación entre profesionales y pacientes (Nieuwwnhuijze, 2013). Las mujeres entrevistadas piden soporte y atención de los profesionales. Hodnett (2002) mostró en una revisión sistemática sobre «Dolor y satisfacción de las mujeres con la experiencia del parto» que eran cuatro los principales factores asociados con la satisfacción del parto: (1) Expectativas personales, (2) la cantidad de apoyo de los cuidadores, (3) la calidad de la relación cuidador-paciente, y (4) la participación en la toma de decisiones. Según 
Hodnett, estos factores parecen ser tan importantes que anulan la influencia de la edad, el estado socioeconómico, el origen étnico, la preparación para el parto, el entorno físico del parto, el dolor, la inmovilidad, la intervención médica y la continuidad de la atención cuando las mujeres evalúan su experiencia en el parto.

\section{CONCLUSIONES}

El proceso de toma de decisiones se presenta como un viaje en el que se va forjando la decisión. Las mujeres no siempre toman decisiones firmes en el momento de cumplimentar el plan de parto; las decisiones tomadas durante el embarazo pueden ser provisionales, simples declaraciones de intenciones. En el momento del parto, el dolor percibido puede vivirse como una seria amenaza frente a la cual se sienten vulnerables. El rol de matronas y otros profesionales sanitarios puede ayudar a la resolución de esta sensación de vulnerabilidad o, por el contrario, constituir una dificultad añadida.

El imaginario colectivo en relación con el afrontamiento del dolor en el parto se ve influenciado por una tendencia social a evitar las sensaciones dolorosas mediante técnicas potentes como la anestesia epidural, que cuenta con una importante tradición en España. Para avanzar hacia un aumento en el número de mujeres que opten por métodos no farmacológicos de alivio de dolor, y que además se sientan satisfechas con ellos, se hace necesario profundizar en modelos de relación asistencial adecuados durante el proceso de toma de decisiones que comprendan buena información y una relación deliberativa.

También se hace necesaria la reflexión sobre la forma de denominar los planes de parto, así como profundizar en la formación ética y comunicativa por parte de las matronas y otros profesionales que atienden embarazo y parto para actuar como expertas consejeras sin caer en un paternalismo sino con auténtico respeto al principio de autonomía de las mujeres en la toma de decisiones. 


\section{BIBLIOGRAFÍA}

Agencia de Calidad del Sistema Nacional de Salud. Observatorio de Salud de LAS MuJERES. Plan de parto y Nacimiento. Estrategia de atención al parto normal, Estrategia Nacional de Salud Sexual y reproductiva. Madrid: Ministerio de Sanidad, Política Social e Igualdad, 2010.

ArAgON, M., et al. Perspectives of expectant women and health care providers on birth plans. En: J Obstet Gynaecol Can. 2010, vol. 35, núm. 11, pp. 979-985.

BRAUER, S. Moral implications of obstetric technologies for pregnancy and motherhood. En: Med Health Care Philos. 2016, vol. 19, núm. 1, pp. 45-54.

BRISCOE, L., et al. A concept analysis of women's vulnerability during pregnancy, birth and the postnatal period. En: Journal of Advanced Nursing. 2016 (junio), pp. 23302344

CoXon K.; J. SAndall; N. J. Fulop. To what extent are women free to choose where to give birth? How discourses of risk, blame and responsibility influence birth place decisions. En: Health, Risk \& Society. 2014, vol. 16, núm. 1, pp. 51-67,

ESCURIET-PEIRO, R., et al. Impact of maternity care policy in Catalonia: a retrospective cross-sectional study of service delivery in public and private hospitals. En: BMC Pregnancy \& Childbirth. 2015, vol. 15, núm. 23.

Generalitat de Catalunya. Departament de Sanitat i Seguretat Social. Protocol d'assistència al part $i$ al puerperi i d'atenció al nadó. Barcelona: Direcció General de Salut Pública, 2003.

GiJón, N. Plan de parto: nomenclatura, toma de decisiones e implicación de los profesionales sanitarios. En: MUSAS: Revista de Investigación en Mujer, Salud y Sociedad. 2016, vol. 1, núm. 2, pp. 35-51.

GobIERnO DE EsPAÑA. JEFATURA DE ESTAdO. Ley 41/2002, De 14 de Noviembre, Básica Reguladora de la Autonomía del Paciente y de Derechos y Obligaciones en Materia de información y documentación clínica. En: BOE. 2002, vol. 274. Disponible en: 〈www.boe.es/buscar/act.php?id=BOE-A-2002-22188>.

HoDnETT, E. D. Pain and women's satisfactions with the experience of childbirth: a systematic review. En: Am J Obstet Gynecol. 2002, vol. 186, núm. 5, pp.160-172.

International Bioethics COMmittee. The Principle of Respect for Human Vulnerability and Personal Integrity: Report of the International Bioethics Committee of UNESCO (IBC). Paris: UNESCO, 2013. 
LALLY, J. E., et al. Pain relief in labour: a qualitative study to determine how to support women to make decisions about pain relief in labour. En: BMC Pregnancy Childbirth. 2014, vol. 6 .

MatuA, G. A. Choosing phenomenology as a guiding philosophy for nursing research. En: Nurse Researcher. 2015, vol. 22, núm. 4, pp. 30-34.

Ministerio De SANIDAD y CONSUMO. Estrategia de atención al Parto Normal en el Sistema Nacional de Salud. Madrid: Ministerio de Sanidad y de Consumo, 2008.

Ministerio de SANIDAd y Política Social. Guía de Práctica Clínica sobre la Atención al Parto Normal. Vitoria-Gasteiz: Servicio Central de Publicaciones del Gobierno Vasco, 2010

NIEUWENHUIJZE, M.; L. K. LOW. Facilitating women's choice in maternity care. En: $J$ Clin Ethics. 2013, vol. 24, núm. 3, pp. 276-282.

RendtorfF, J. D. y P. KeMP. Basic Ethical Principles in European Bioethics and Biolaw (Vol I: Autonomy, Dignity, Integrity and Vulnerability). Barcelona: Center for Ethics and Law, Copenhague - Instituto Borja de Bioética, 2000.

SABATÉ, S., et al. Anestesia obstétrica en Cataluña. En: Medicina Clínica. 2006, vol. 126, núm. 2, pp. 40-5.

TAYLOR, S. J.; R. BOGDAN. Introducción a los métodos cualitativos de investigación. La búsqueda de significados. L'Hospitalet de Llobregat: Paidós, 1987.

VAN DER GUCHT, N.; K. LEWIS. Women's experiences of coping with pain during childbirth: A critical review of qualitative research. En: Midwifery. 2015, vol. 31, núm. 3, pp. 349-358.

WELSH, J.; A. G. SYMON. Unique and proforma birth plans: A qualitative exploration of midwives' experiences. En: Midwifery. 2015, vol. 30, núm. 7, pp. 885-891.

WhitFORD, H., et al. Use of a birth plan within woman held maternity records: A qualitative study with women and staff in Northeast Scotland. Birth. 2014, vol. 41, núm. 3, pp. 283-289. 